\title{
System of information output to electronic maps about vessels and aircrafts movement
}

\author{
Vitaly Vergeichik ${ }^{1}$, Vadim Kramar $^{1, *}$, Veronika Dushko $^{2}$, and Andrey Volodin ${ }^{1}$ \\ ${ }^{1}$ Nakhimov Black Sea Higher Naval School, 299035 Dibenko st., 1, Sevastopol, Russian Federation \\ ${ }^{2}$ Sevastopol State University, 299053 Universitetskaya st., 33, Sevastopol, Russian Federation
}

\begin{abstract}
The article is devoted to actual problem of development and introduction of the replay system of the current location and direction of movement, in real time vessels and aircrafts, intended for continuous monitoring and obtaining data on the characteristics of vessels.
\end{abstract}

\section{Introduction}

Modern technology has caused the increase of technological advancements.

In the past few years it has been seen that the following questions are put in first place: data support, which helps to achieve superiority, real-time information exchange, adjustments of the ship's movements at sea [1].

The research is based on the problem of creation of a possibilities modelling system, which will also aim to technically solve the information field for information reflection via various industrial gauges with a trajectory measurements complex register [3].

At the present time, control of the situation in the area is carried out from various display devices, which cannot ensure that security control is provided to the full extent.

\section{Main part}

Computer programs used in telemetry are significantly different from those used in less critical applications. Since the telemetry data arrives at the receiving station repeatedly and sometimes even continuously, the hardware and software must be well coordinated with each other. In typical cases, hardware works relatively simple and repetitive tasks (for example, the establishment of synchronization and response to the emergence of an alarming situation); The software performs a primary processing to reproduce the data on the screen. The tasks of the software include setting up all hardware and software, high-speed data entry, possible preliminary hardware testing, high-speed output of specially selected measurement results to display terminals, display formatting and special data processing in accordance with the analysis requirements. The software is also often used to prepare a disk drive to work with all or selected measurement results to retrieve data from the disk for more detailed analysis and to perform a self-diagnosis of the state of the telemetry system before and during data reception.
As a means of operational control [2], each individual electronic map exists only at a certain point of time, as a rule, a short one, while it is visible on a display device. This is their main difference from other visual cartographic documents visualized on a solid surface (paper, plastic) by the means of graphic output (printers, for example).

This meaning agrees best with the word "electronic" [7], i.e. obtained through the movement of electrons, which is what happens in a working electronic device.

In accordance with the standard, a digital terrain model is a logical mathematical representation of the topographic survey data about the objects of the terrain, its characteristics, and includes:

- metric information - geodetic spatial data of the distinguished points of the terrain and situation;

- syntactic information to describe the connections between the points - borders of the buildings, forests, plowlands, water reservoirs, roads, watershed and overflow lines, slope direction between the characteristic points on the slopes, etc.;

- semantic information that characterizes the properties of objects - technical parameters of engineering structures, geological characteristics of the soil, information about the trees in forest areas, etc.;

- structural information that describes the relationship between different objects - the relationship of objects to any set: separate points of the railway line, buildings and constructions of a settlement, buildings and structures of the relevant facilities, etc.;

- general information - name of the site, system of coordinates and heights, nomenclature.

Topographic digital terrain model characterizes the condition and lay of the ground. It consists of a digital terrain model and digital model outline of the (situation) area. In addition, a digital terrain model can be supplemented with a special-purpose engineering model. When it comes to the engineering practice, a combination of digital models is often used; the models feature the situation, terrain, hydrological, engineering, geological, technical, economic and other indicators.

*Corresponding author: kramarv@mail.ru 
In seeking to resolve the geodetic engineering problems on a personal computer (PC), a mathematical interpretation of digital models is used, which is called a mathematical terrain model. Computer-assisted design, which is based on a digital terrain model and a mathematical terrain model, reduces labor and time expenditure by the dozens of times, compared to the use of paper topographic maps and plans for this purpose.

In this regard, the electronic map symbolization system includes special fonts, and the classification of the electronic maps corresponds to the general map classification, for example: electronic topographic map, electronic aviation map, electronic geological map, electronic cadastral map, and others.

A digital map is a digital cartographic model, the content of which corresponds to the content of the map of a certain type and size. The digital map is used for the manufacture of paper and electronic maps, it is included in a map database, and it represents a geodetic topographic background for geographic information systems.

An electronic map is a digital cartographic model, visualized and prepared for visualization on the screen of a display device for presentation of information in a special system of symbols, the content of which corresponds to the content of a map of a certain type and size.

The advantages of electronic maps are listed below:

- possibility to change it as soon as new data are processed;

- automatic search and delivery of the information required;

- scaling of an image;

- layered data display, i.e. only of the information the user needs.
The Sirius software (certificate of state registration of computer programs No.2016662511 dated November 11th, 2016) [6] is designed to collect, display and predict the situation in the area with the construction of moving objects trajectory according to the data from a radar; it also helps to control and monitor the air and surface picture for security purposes.

PC with a recording device for trajectory measurements provides a continuous origination of actual real-time coordinates of a product, which is enough to identify it, regardless of geographic application conditions, weather conditions; it also records the instrument errors when determining the angle of azimuth and slant visual range (fig. 1).

In addition, when it comes to determining the characteristics of a product, the software improves the productivity and lowers the cost of production.

The use of electronic maps of the area allows one to outline the air and maritime situation; a block scheme of a hardware component is shown in picture No.1. Data from a radar station reveal the location of ships and unidentified targets; the software displays the coordinates, calculates the speed and direction of a tracked object, indicates the time when the object enters/leaves the area. The air picture is visualized in real-time.

A real-time information output on the electronic map from different technical elements allows one to control the area and, in case of security violation, make decisions concerning the termination of the motion of a sea vessel/aircraft.

It is also possible to save data entered by the user, as well as to open saved files.

On completion of simulation, an output file is being created; it can be used to check real devices for signal processing.

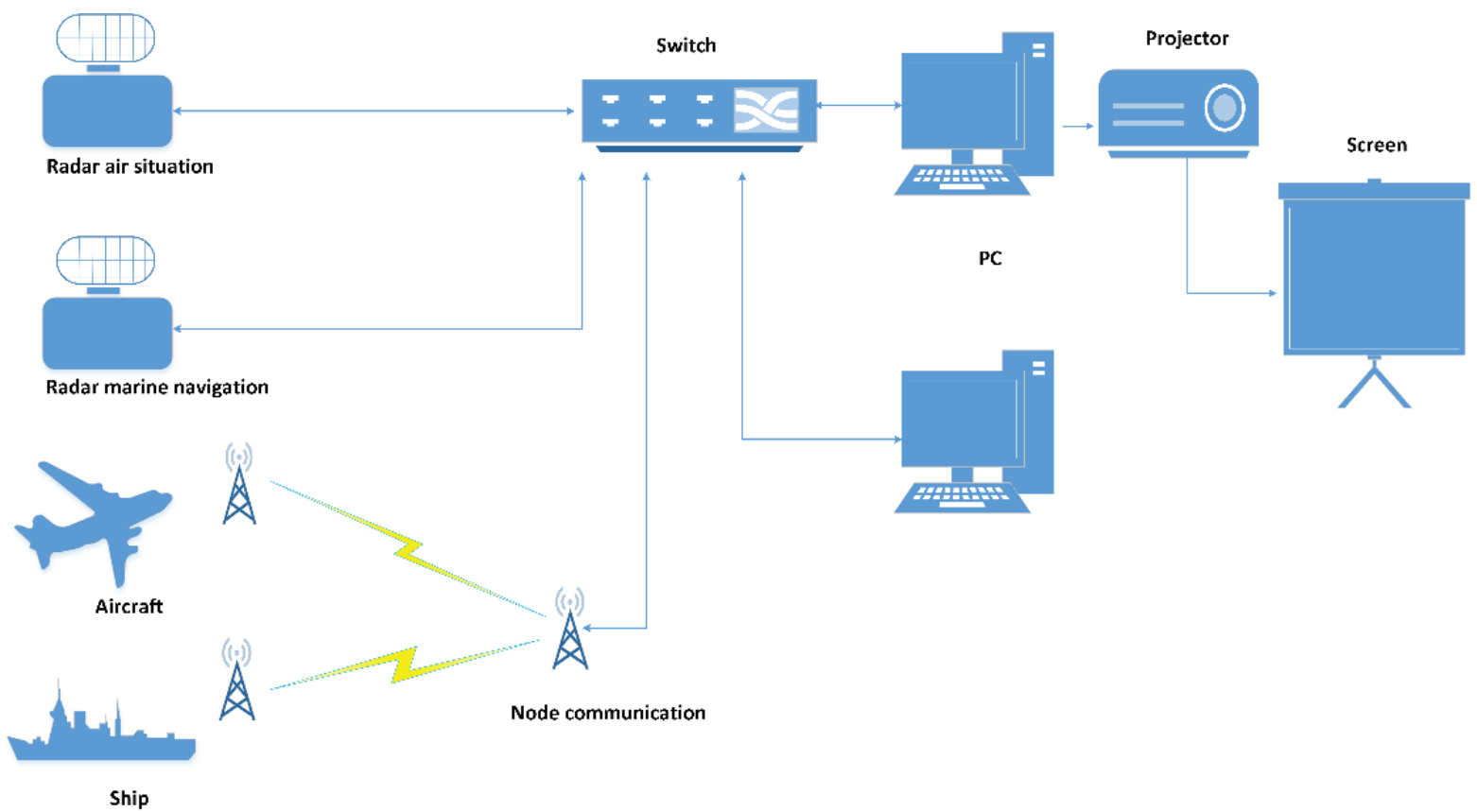

Fig.1. Oscillograms for the seaworthiness evaluation problem 
Data is imported from the files with a radio-locating situation to the real devices for signal processing.

Determination of available hardware resources, search for drivers for these devices, as well as their initialization, take place at initializing the hardware and software. Data exchange with a limited-purpose device is organized under the control of a previously selected driver.

Data exchange with other applications is organized, the exchange takes the form of graphic information and information that may be used to organize the process of simulation, by the results of simulation itself.

The simulation of a radio-locating situation, based on the data provided by the user, calculates the signals which form a radio-locating situation, as well as converts the curvilinear coordinates system into the Gauss-Kruger plane rectangular coordinates system.

Capability research was carried out, theoretical solutions concerning the way to transfer the information data and technical ways of the information field on the display of information from different technical elements was found.

The software reproduces the current position and direction, in real time [4], as well as displays the most important parameters for control and comparison with the standards in a rapid analysis mode, by means of communication channels it can also transmit digital information to the closely-spaced areas [5].

The following methods have been used at completing general and particular tasks of the research: the methods of matrix theory, linear algebra, curvilinear coordinates system and Gauss-Kruger plane rectangular coordinates system, computer generated simulation. Computer generated simulation was performed with the help of a mathematics software, $\mathrm{C}++$ Builder 6.0. and the use of matrix algebra numerical computing.

The Sirius software family (fig. 2) solves tasks at three different levels:

- a registration level;

- a collection rate;

- a display level.
Software main features:

- receiving and decoding of information from a radar in real scale;

- calculation of the geographical values of the object tracked by a radar;

- calculation of the distance to the object;

- multicolored visualization of the trajectory of a tracked object on a digital map, with the color depending on the radar tracking quality of the object;

- detailed decoding, display of radar operation, chart output;

- obtainment and display of target indications from a radar;

- collection of objective situation from a radar;

- display of the radar tracking quality of each object;

- the choice of radar, which will calculate the indications;

- display of trajectory and radar tracking quality;

- calculation of the speed and direction of the target movement;

- display of the history sheet and trajectory of an object on a digital map;

- calculation of the time when the object enters/leaves the area.

Digital data can be converted to different formats, as well as transformed from one form to another, it can be visualized on graphic displays, printed, edited, changed, etc.

Electronic map classifier is an independent library of symbols, which contains all possible graphic descriptions of objects.

The external object code, layer, symbol and other characteristics are specified in the description of an object. However, for application of any additional graphic information (descriptive information, split lines, polygons, etc.) it is more convenient to use random graphic primitives without their prior description in a classifier.

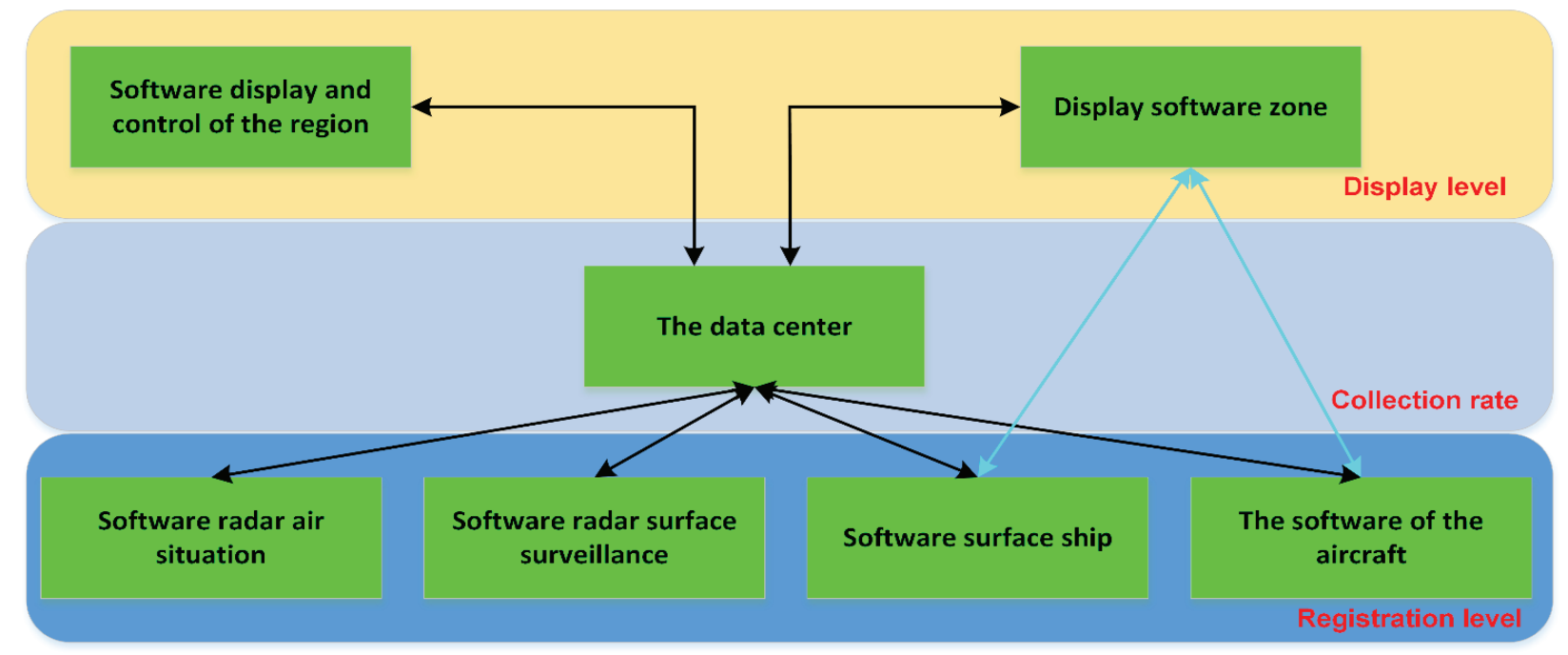

Fig.2 Software architecture of display, collection and recording of information collection Sirius 


\section{Development of mathematical support}

Currently, the modeling of various processes and phenomena is widely used for a variety of research and development in various fields of science and technology. The use of modern computer technology makes it possible to obtain sufficiently accurate models that accurately describe the simulated phenomenon or process. The most widely used mathematical and simulation modeling.

At the heart of mathematical modeling are mathematical models - every process or phenomenon is described by mathematical equations, which requires a serious mathematical apparatus. Implementation of mathematical models on a PC is possible when using numerical methods to implement various mathematical functions and operators, which introduces an error in the calculations.

Simulation modeling involves obtaining and using a model that describes the mechanics of a process or phenomenon, i.e. How the process proceeds in the real situation and what underlies this process. Simulation modeling, in general, relies on physical laws, for the implementation of which the device of mathematical functions is also needed.

The most acceptable way to describe a simulated domain in this paper is to use simulation modeling based on mathematical models. As such an object, one can take a point target, and divide the entire radar model into models of its main modules. In this case, a compromise will be reached between the accuracy of the model and the total computational cost of implementing the model.

\section{Conclusions}

Therefore, the system of registration, information processing will ensure the increase of security and visibility level, it will also reduce the time of processing and documentation of the results. That will not only comply with all the requirements for the information transfer, but will also reduce the cost of maintenance of the telecommunications infrastructure.

The reliability of the results is confirmed by experimental verification of performance of the developed algorithms, which have confirmed the improved accuracy, in comparison with analogues.

A new technical solution for area monitoring has been found. The software reproduces the current position and direction, in real time, as well as displays the most important parameters for control and comparison with the standards in a rapid analysis mode, by means of communication channels it can also transmit digital information to the closely-spaced areas.

\section{References}

1. V. V. Vergeichik,. V. V. Buldakov, Purpose and performance characteristics of promising measurement tools during the field works, Scientific readings on aviation dedicated to the memory of $\mathrm{N}$. E. Zhukovsky, No.4, Pp.361-370 (2016)

http://elibrary.ru/item.asp.2id=26570905

2. D. Yu. Polenov, The Evolution of telemetry in rocketry, Young scientist, No.6, Pp. 216-218 (2014)

3. A. A. Guzinski, V. V. Vergeichik, V. A. Kramar, Increase of efficiency of use of measuring instruments for the Navy, Innovative Arsenal youth: proceedings of the seventh scientific - technical conference of young scientists and specialists of FSUE "KB Arsenal", issue No.7, Pp. 295-298 (2016)

4. V. I. Pan-Luzin, Telemetry. The eyes and ears of the Chief designer (Moscow: OOO "Overlay", 2009)

5. A. V. Nazarov, Modern telemetry in theory and practice (St. Petersburg: Science and technology, 2007)

6. A. A. Guzinski, O. V. Hletko, V. V. Vergeichik, Collection, records and displays the trajectory of the product on an electronic map in real time according to the radar data and telemetry data, control and monitoring of surface and air situation in the area of the experiment to ensure security, Certificate of state registration of computer programs №2016662511, date of the state registration in the Register of the Federal service for intellectual property (ROSPATENT) computer programs November 11, 2016

7. The

Internet: http://dic.academic.ru/dic.nsf/ruwiki/70403 0 (accessed 22.10.2016), " Electronic map. 